\title{
Uncommon presentation of giant cell arteritis: Report of two cases with scalp necrosis
}

Thomas M ücke, M arco R. Kesting, Frank H ölzle, Klaus-D ietrich W olff

Department of Oral and Maxillofacial Plastic Surgery, Klinikum Rechts der Isar der Technischen Universität München, Germany

\begin{abstract}
Giant cell arteritis (GCA), the most common form of systemic granulomatous vasculitis in adults, preferentially involves large and medium-sized arteries. Scalp necrosis is a rare complication and can be the presenting feature. This report presents two patients of GCA with severe scalp necrosis as the presenting symptoms. Both the patients were treated successfully with selective debridement, local wound care, antimicrobial and systemic steroid treatment. Skin necrosis is a potentially treatable complication of GCA and needs a multidisciplinary approach.
\end{abstract}

Address for correspondence:

Dr. Thomas M ücke,

Department of Oral and

Maxillofacial Plastic Surgery, Klinikum

Rechts der Isar der Technischen

Universität München, Ismaninger

Str. 22, 81675 M ünchen, Germany.

E-mail: th.mucke@gmx.de

DOI: $10.4103 / 0028-3886.48817$
Key words: G iant cell arteritis, infarction, necrosis, surgery, systemic disease, wound management

\section{Introduction}

Giant cell arteritis (GCA) is a disease with typical histopathologic findings which may be panarteritis and it may be granulomatous or lymphocytic arteritis, Arterial lumen may be compromised by arterial wall destruction and edematous swelling of the intima. ${ }^{[1,2]}$ Superficial temporal, vertebral, ophthalmic and posterior ciliary arteries are commonly affected in GCA ${ }^{[1]}$ Rarely in about $1 \%$ it can be generalized, particularly in the elderly. Patients with GCA more often present with systemic, ophthalmic, and neurological symptoms. ${ }^{[2,3]}$ This paper presents two patients of GCA with scalp necrosis, an uncommon presenting feature.

\section{Case Reports}

\section{Case 1}

A 81-year-old man presented with painful scalp lesions, scalp hypersensitivity and headache. Past medical history included: amaurosis of the right eye, myocardial infarction and heart failure, hypertenson, carotid stenosis, ischemic stroke, dementia and chronic kidney disease. The scalp lesions were initially minor but at presentation were more extensive, necrotic and infected [Figure 1a, Figure 1b]. The temporal arteries were tender and non-pulsatile. Erythrocytes sedimentation rate (ESR) was $90 \mathrm{~mm}$ at first hour and C-reactive protein (CRP) was $9.26 \mathrm{mg} / \mathrm{dl}$. With the diagnosis of GCA, confirmed by arterial biopsy the patient was started on oral prednisolone $60 \mathrm{mg}$ per day. Over the next few days there was extension of the scalp necrosis on both the sides and patient experienced a great deal of pain. In addition to systemic steroids, he had debridement of the wound without the use of vasoconstrictors and local anesthesia, local wound care (paraffin gauze dressing, Jelonet ${ }^{\circledR}$, Smith and Nephew, Germany), and antibiotics. After two months, prednisolone dose was reduced gradually to $5 \mathrm{mg}$ daily. The scalp lesions healed and the patient had improved general condition. In this patients it is quite possible some of the medical problems which he had like stroke, peripheral vascular disease, stroke, carotid stenosis, and myocardial infarction might have been related to GCA. We feel probably this patients had generalized systemic GCA.

\section{Case 2}

A 87-year-old woman presented with an abrupt onset severe scalp pain, scalp hypersensitivity, vertigo and malaise. Clinical examination revealed extensive dark necrotic lesions on both the parieto-occipital scalp regions [Figure 1c, Figure 1d]. Both the temporal arteries 

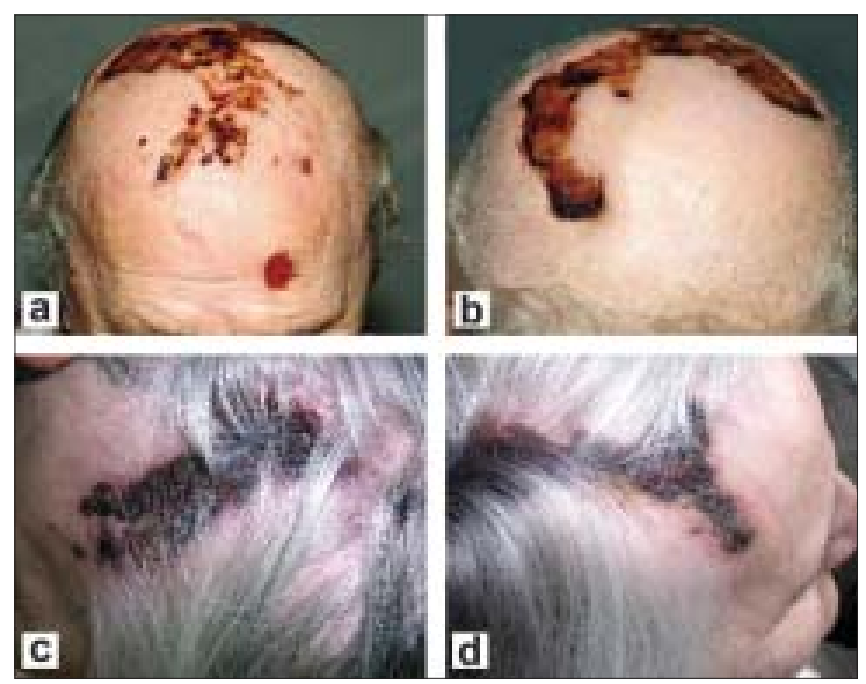

Figure 1 a-d: Patients suffering from scalp necrosis. Patient $1(a, b)$ had an infection of the necrotic tissue, which had to be treated surgically, whereas patient $\mathbf{2}$ (c, d) was treated conservatively

were feeble but non-tender. There was no evidence of infection of the lesions. ESR and CRP measurements were $43 \mathrm{~mm}$ at first hour and $2.3 \mathrm{mg} / \mathrm{dl}$ respectively. Diagnosis of GCA was confirmed by biopsy and patient was started on prednisolone $60 \mathrm{mg}$ daily. Over the next few days the general condition showed a rapid improvement and the vertigo symptom resolved. Scalp necrosis on the side contralateral to the biopsy side became more extensive. In this patient there was no need for wound management or dressing. At the time of discharge the scalp wounds were healthy. After one month, the dose of prednisolone was reduced gradually to $8 \mathrm{mg}$ daily. She still under follow-up.

\section{Discussion}

The first description of scalp necrosis in GCA was by Cooke et al. ${ }^{[4]}$ Since then, about 30 cases have been reported. ${ }^{[5]}$ GCA, also called "arteritis of the aged, rarely manifests under the age of 60 years. Clinical manifestations of GCA are protean. Rare but severe complications are skin, scalp or tongue necrosis in varying degrees of severity ${ }^{[6]}$ which in some cases need to be treated surgically. ${ }^{[7]}$ Brainstem strokes and dementia have also been described ${ }^{[3,8]}$ and coronary artery involvement is possible. ${ }^{[9]}$ Most often the diagnosis is obvious but in patients with uncommon clinical presentation the diagnosis may be difficult. ${ }^{[8]}$ Furthermore, in some patients it becomes difficult to attribute the vascular events to GCS or atherosclerosi. There has been no proven association between increased incidence of myocardial infarction, stroke and/or dementia and GCA. ${ }^{[8]}$

Clinically, GCA-associated skin necrosis might be misdiagnosed as erosive pustular dermatosis, more so when artiritis involves one of the branches of temporal artery. This entity is characterized by a phasedchronic course. Other differential diagnoses include skin infections. Unilesional pyoderma gangrenosum of the scalp represents a rare complication, which is more frequently described in male than in female patients. Also Wegener's granulomatosis can lead to skin ulcerations.

GCA is a common and important disease and involves almost all the specialties and is associated with high morbidity and mortality if not diagnosed and treated early. ${ }^{[10]}$ Thromus formation followed by intra-arterial embolism within the inflamed vessels can affect all organs. ${ }^{[2,6-9]}$ These complications should be treated with an interdisciplinary concept of managing the consequences of the disease. ${ }^{[10]}$

Our concept of wound management include: (1) immediate institution of high dose corticosteroid treatment. (2) local and systemic treatment of the infection by desiccating or keeping wounds dry, and antibiotics; (3) debridement of the wound without the use of vasoconstrictive agents or local anesthesia if possible to avoid additional ischemia. We found this approach helps in early healing of the wound, this is seen even with extensive necrotic lesions. Early diagnosis and treatment with corticosteroids can prevent severe complications of GCA, both systemic and local.

\section{References}

1. Siemssen S.J. On the occurrence of necrotising lesions in arteritis temporalis: Review of the literature with a note on the potential risk of a biopsy. Br J Plast Surg 1987;40:73-82.

2. Weyand CM, Goronzy J.J. Medium-and large-vessel vasculitis. N Engl J Med 2003;349:160-9.

3. Levine SM, Hellmann DB. Giant cell arteritis. Curr Opin Rheumatol 2002;14:3-10.

4. Cooke WT, Cloake PC, Govan AD, Colbeck JC. Temporal arteritis: A generalised vascular disease. Q J Med 1946;15:47-75.

5. Currey J. Scalp necrosis in giant cell arteritis and review of the literature. Br J Rheumatol 1997;36:814-6.

6. Morris OC, Paine MA, O'Day J. Giant cell arteritis presenting with scalp necrosis--the timing of temporal artery biopsy? Clin Exp Ophthalmol 2006;34:715-6.

7. Biebl MO, Hugl B, Posch L, Tzankov A, Weber F, Perkmann R, et al. Subtotal tongue necrosis in delayed diagnosed giant-cell arteritis: A case report. Am J Otolaryngol 2004;25:438-41.

8. Morris OC, Lockie P. Giant cell arteritis-presenting as stroke, transient ischaemic attack and dementia. Aust Fam Physician 2005;34:653-5.

9. Paulley JW. Coronary ischaemia and occlusion in giant cell (temporal) arteritis. Acta Med Scand 1980;208:257-63.

10. Nordborg E, Nordborg C. Giant cell arteritis: Epidemiological clues to its pathogenesis and an update on its treatment. Rheumatology (Oxford) 2003;42:413-21.

Accepted on 05-02-2009

Source of Support: Nil, Conflict of Interest: None declared. 